\title{
I FATTORI DELLA VIRTÙ E LA CRONOLOGIA DELLE ETICHE DI ARISTOTELE
}

\author{
Nota del s.c. PIERLUIGI DONINI (*)
}

(Adunanza del 30 gennaio 2014)

SuNTO. - In questo saggio l'autore riassume le principali tesi che aveva esposto nel suo nuovo libro Abitudine e saggezza. Aristotele dall'Etica Eudemia all'Etica Nicomachea. Nell'Eudemia Aristotele presenta la virtù come il risultato dell'interazione di ragione e doti di natura, mentre nella Nicomachea non nomina affatto la natura e vede la virtù come il prodotto delle abitudini e dell'educazione. Proprio a questo proposito l'EN cita con approvazione le Leggi di Platone, che erano ancora ignote ad Aristotele quando egli componeva l'EE. Sembra evidente che nel tempo trascorso tra la composizione delle due Etiche Aristotele aveva avuto conoscenza dell'ultima opera di Platone.

\section{$* * *$}

ABSTRACT. - In this paper the author summarizes the main contentions made in his new book Abitudine e saggezza. Aristotele dall'Etica Eudemia all'Etica Nicomachea. In the Eudemian Ethics Aristotle makes moral virtue as the joint effect of reason and the gifts of nature, but in the Nicomachean Ethics, on the contrary, he does not even name nature and sees virtue as the product of habits and education. On this very point the $N E$ quotes and praises Plato's Laws, which Aristotle did not know when writing the EE. It seems clear, then, that in the interval between the two Ethics he had known Plato's last work.

(*) Università degli Studi di Milano, Italia. E-mail: pierdonini@alice.it 
1. Le Etiche di Aristotele sono ancora, per la storiografia filosofica, un problema molto controverso. ${ }^{1}$ Dall'antichità, sotto il nome del filosofo, ne sono giunte ben quattro. Ma di due, i Magna Moralia (o Grande Etica) e il libretto Sulle virtù e i vizi, non occorre dire nulla: non sono opere autentiche e il discorso si può chiudere qui. Ne rimangono due, riconosciute ormai universalmente come autentiche, la celeberrima Etica Nicomachea e l'Etica Eudemia, in età moderna da sempre rimasta nell'ombra e schiacciata dal confronto con la Nicomachea. Gli storici e i filologi, ma direi anche i filosofi, non possono però fare a meno di chiedersi quale delle due presenti il punto di vista definitivo di Aristotele e - soprattutto - perché egli sentì il bisogno di scrivere due esposizioni dello stesso argomento. Dopo circa due secoli di discussioni, le risposte non sono affatto unanimi. C'è sì una certa maggioranza di studiosi che considera l'Eudemia anteriore alla Nicomachea, ma continuano ogni tanto a insorgere proposte di invertire il rapporto cronologico (l'ultimo caso, recentissimo, proprio in Italia). ${ }^{2}$ Quanto al perché della composizione di due opere sullo stesso argomento, questa è una domanda che non viene oggi tanto spesso posta. Prevale infatti una sorta di interpretazione concordistica che tende a dare rilievo più alle cose che le Etiche avrebbero in comune che a quelle che potrebbero differenziarle. Ma ci sono anche alcuni tentativi (ai quali non voglio nascondere che va la mia simpatia) di rispondere invece proprio al problema di saper distinguere bene le due Etiche dal punto di vista delle dottrine e di spiegare così perché Aristotele avrebbe sentito il bisogno di riscrivere la sua prima etica, quale che questa sia.

2. Credo opportuno muovere dal problema assolutamente preliminare. Se la questione più interessante è quella di capire perché Aristotele sentì la necessità di trattare una seconda volta il medesimo tema, quale che sia la più antica e quale la più matura delle due opere, sarebbe tuttavia bene aver chiaro in partenza quale delle due si debba considerare la più

1 In questa breve nota riassumo l'essenziale dell'argomentazione che ho svolta nel libro Abitudine e saggezza: Aristotele dall'Etica Eudemia all'Etica Nicomachea, Alessandria, Edizioni dell'orso 2014.

2 Mi riferisco all'edizione curata da Marcello Zanatta di Aristotele, Etica Eudemia, Milano, RCS libri 2012.

3 Penso soprattutto alla recente edizione inglese curata da Brad Inwood e Raphael Woolf, Aristotle, Eudemian Ethics, Cambridge, UP 2013. 
antica e quale la più recente. Ma oggi ha (credo) una certa prevalenza negli studi aristotelici l'idea che non ci sono argomenti obiettivi per risolvere questo problema e c'è, inoltre, una giusta diffidenza verso i criteri che per risolvere la questione furono utilizzati in passato (e alcuni ancora li utilizzano oggi) e che non è necessario qui ricordare nei dettagli. Mi limiterei a dire che non è certamente un buon criterio quello, abbastanza diffusamente impiegato, che assuma che deve essere posteriore l'opera che all'interprete di oggi appare la "migliore" - migliore perché sarebbe più profon$\mathrm{da}$, più completa, più coerente, più ricca, più bella: i parametri che così si impiegano (completezza, coerenza, profondità, bellezza) sono infatti troppo soggettivi e personali, peculiari per ciascun interprete, per risultare obiettivamente affidabili. Che fare, allora? Di solito si dice anche che non esistono nelle due Etiche dati obiettivi (p. es. il riferimento a fatti storici sicuramente databili) che permettano di collocarle con sicurezza nel tempo. Ma questo non è del tutto vero, come cercherò di spiegare; è vero però che sul dato di fatto che come tale citerò bisogna ancora lavorare con pazienza e capacità di interpretazione storico-filosofica.

3. Questo dato, questo fatto da cui io partirei è una citazione esplicita che ricorre nell'Etica Nicomachea e solo in questa, mai nell'Eudemia, delle Leggi di Platone, che sappiamo con certezza da altre testimonianze antiche (p.es. lo stesso Aristotele in Pol. II 6, 1264b25) essere l'ultima opera di Platone, da lui lasciata incompiuta. Dunque la Nicomachea è posteriore alle Leggi e alla scomparsa di Platone (cioè all'anno 348). Ma di qui a concludere che allora anteriore a quella data dovrebbe essere l'Eudemia, che non cita mai le Leggi, evidentemente ce ne corre: si possono immaginare decine di ragioni per cui in un'altra opera anch'essa posteriore al 348 Aristotele avrebbe potuto decidere di non parlare più delle Leggi. In sé, dunque, la citazione dell'EN non dice nulla e, per quanto ne so, non è infatti mai stata sfruttata a fondo al fine di definire la relazione tra le due Etiche.

$\mathrm{Ma}$ ci si può lavorare sopra meglio. Non è infatti la citazione di un passo qualsiasi, ma di uno che risulta centrale nell'illustrazione di un punto di dottrina che sarà fondamentale nella Nicomachea ed è invece sostanzialmente assente nell'Eudemia. Aristotele cita Platone, infatti, per corroborare la propria esposizione relativa al modo di formazione della virtù morale nel II libro dell'EN: dove espone la dottrina (celebre, credo, e nota anche dal solo insegnamento della storia della filosofia nei licei) - la dottrina che fa nascere la virtù morale dalle buone abitudini e 
dall'assuefazione ad agire in modo buono: si diventa coraggiosi abituandosi ad affrontare i pericoli, giusti abituandosi a praticare la giustizia, temperanti a ripetere atti di temperanza e così via. Ora, di questa sua tesi Aristotele trova una conferma appunto nell'ultima opera del suo maestro e lo cita con approvazione esplicita quasi all'inizio del secondo libro dell'EN:4 "perciò - dice - bisogna in certo modo essere guidati subito, fin da bambini, a gioire e soffrire delle cose di cui si deve [cioè ad aver piacere delle azioni buone proprie e altrui, a soffrire di quelle cattive, cioè a provare per esse avversione e ripugnanza], come dice Platone. Questa è infatti la retta educazione". L'accenno alla "retta educazione" combinato con quello al "gioire e soffrire per le cose di cui si deve" ci indicano con precisione persino la pagina delle Leggi che Aristotele aveva in mente, cioè un testo del secondo libro, 653a-c, un passo che non posso fare a meno di citare anche perché può servire come un ottimo compendio della teoria dello stesso Aristotele nell'EN, salvo pochi particolari non importantissimi in questa sede:

affermo dunque che nei fanciulli la prima sensazione puerile sono il piacere e il dolore e che è nel loro ambito che virtù e vizio per la prima volta compaiono nell'anima; mentre l'intelligenza e le stabili opinioni rette è fortunato colui cui esse giungano anche in prossimità della vecchiaia: ed è un uomo compiuto colui che ha acquisito queste doti e tutti i beni che esse racchiudono. Dico "educazione" la virtù che per prima perviene ai fanciulli; il piacere e l'affezione e il dolore e l'avversione, se nascano correttamente nelle anime che ancora non possono ospitare la ragione e, una volta acquistata la ragione, concordino con questa riconoscendo di essere stati rettamente abituati dalle abitudini convenienti - questa concordia è nel suo complesso la virtù; ma quella parte di essa che è relativa ai piaceri e ai dolori e che sia stata correttamente allevata, sì da odiare quel che si deve odiare fin dal principio e fino alla fine e amare quel che bisogna amare, questa parte, distinguendola dalla ragione e chiamandola educazione a mio avviso sarebbe correttamente denominata.

E' questa proprio la stessa teoria che Aristotele espone e difende nella prima metà del libro II della Nicomachea, salvo il fatto che non dà il nome di "educazione" alla virtù formata dalle sole abitudini; per tutto il resto si potrebbe dire che egli rimane perfettamente platonico (del platonismo delle Leggi) proponendo e difendendo le ragioni di una virtù che nascerebbe da un'educazione delle pulsioni fondamentali di

4 II 3, 1104b11-13. 
piacere e dolore le quali, mediante l'assuefazione delle abitudini, andando oltre il soddisfacimento delle esigenze elementari della vita biologica (cibo, riparo, sicurezza), verrebbero indirizzate verso il piacere della virtù e l'avversione alla malvagità, fino al sorgere di una razionalità che non farebbe altro che concordare con le abitudini fino ad allora assorbite. Bene: di questa teoria che può piacere o no, ma è la teoria tipicamente aristotelica della virtù che è anche oggi esposta nei nostri manuali ed è, in realtà, una teoria ancora largamente platonica, nell'Etica Eudemia non c'è quasi niente. Non c'è quasi, in questa versione dell'etica aristotelica, l'abitudine: che è nominata solo cursoriamente due volte (due volte ${ }^{5}$ in oltre 100 pagine di un'edizione critica), non è mai analizzata nel modo che, seguendo Platone, Aristotele illustra nell'EN per metà almeno del secondo libro e su cui, ovviamente, ritorna più volte nel seguito dell'opera (mentre l'EE non ci ritorna mai). Non c'è nemmeno, nell' $E E$, alcun cenno al risultato positivo che l'educazione mediante le abitudini conseguirebbe insegnando a provar piacere della virtù e avversione (dolore) per il vizio, come vogliono la Nicomachea e le Leggi. Che cosa troviamo, in luogo di tutto ciò?

4. Nell'EE c'è una teoria in cui, invece della centralità del fattore "abitudine", compaiono le doti naturali che una volta, alla fine del libro terzo, ${ }^{6}$ Aristotele giunge addirittura a chiamare "virtù naturali", vale a dire buone inclinazioni innate a quegli stati del carattere che, completati poi dalla razionalità, saranno le virtù etiche compiute, giustizia, coraggio, moderazione, temperanza ecc. Le virtù naturali sono invece del tutto ignote all'EN, mai nominate, mai presenti in essa. (Qui però c'è una complicazione a cui non posso non accennare. Chi ha letto con attenzione la Nicomachea mi obietterebbe con ragione che in realtà nel VI e nel VII libro di quest'opera, così come è stampata nelle nostre edizioni, si parla più volte di “virtù naturali”. E' vero, ma c'è qui una trappola da cui guardarsi. Il V, il VI e il VII libro dell'EN figurano anche, nella tradizione manoscritta dei codici, ${ }^{7}$ come IV, V e VI libro dell'EE

5 In EE I 1, 1214a20-21 e II 2, 1220 b1.

6 In III 7, 1224a27-28.

7 Siano poi effettivamente trascritti o no: in alcuni codici la trascrizione non c'è e compare soltanto l'indicazione che il testo di questi libri è identico a quello dei corrispondenti libri dell'altra etica. 
e sono infatti di solito ricordati dagli studiosi come i "libri comuni". Da sempre si discute a quale delle due Etiche dovrebbero in realtà essere attribuiti e oggi si va gradatamente diffondendo la convinzione che essi siano originari dell'Eudemia e poi, per qualche ragione che non siamo in grado di indovinare, siano stati trasferiti nella Nicomachea (forse perché i libri di questa corrispondenti per l'argomento si erano perduti, forse perché Aristotele non li scrisse: non sappiamo). L'argomento principale per aggiudicare questi libri all'EE è stato, ad oggi, di carattere linguistico e stilistico: mediante lo studio statistico di certe espressioni (uso delle preposizioni e delle congiunzioni, nessi linguistici, modi e tempi dei verbi), ${ }^{8}$ uno studioso inglese dimostrò qualche decennio fa che lo stile e la lingua dei libri comuni sono molto più vicini a quelli dell'EE che non all'EN. Personalmente, e con il conforto dell'eguale parere di alcuni altri eminenti aristotelisti, io dò molto credito a indagini di questo tipo, statistico e matematico-quantitativo, aliene da valutazioni di carattere soggettivo; e lo studio comparato delle Etiche e dei libri comuni conferma poi a mio avviso che anche nel contenuto dottrinale e filosofico c'è una maggiore (molto maggiore) affinità tra i libri comuni e l'EE, non con l'EN. ${ }^{9}$ La questione delle virtù naturali e l'assenza quasi completa del fattore "abitudine" nei libri comuni è precisamente uno dei problemi di grande rilievo dottrinale nei quali l'affinità tra quei libri e l'EE va ben oltre il mero fatto linguistico e stilistico. Insomma: rimarrebbe assolutamente vero che l'EN non conosce - o, se è posteriore all' $E E$, non riconosce più - le virtù naturali.

Dunque, riprendendo il filo principale del discorso, mentre l'EN, concordando con il Platone delle Leggi, vede la virtù morale come il risultato dell'educazione mediante le abitudini buone che, a un certo punto della maturazione psichica dei giovani, si sposerebbero all'avvento della razionalità, l'EE la concepisce invece essenzialmente come l'interazione della razionalità con le doti di un carattere che sarebbero innate e naturali. Direi che c'è una bella differenza tra le due concezioni e penso che su questo punto si possa insistere per venire a capo dei problemi, sia quello della cronologia relativa delle opere, sia quello, molto

8 Anthony Kenny, The Aristotelian Ethics, Oxford, Clarendon Press 1978 e Aristotle on the Perfect Life, Oxford, Clarendon Press 1992.

9 Per la dimostrazione di questa tesi mi permetto di rinviare al cap. 5, pp. 139188 del libro citato sopra, nella nota 1 . 
più interessante e importante per la storia della filosofia, del perché Aristotele cambiò idea circa la formazione della virtù e in quale senso la cambiò, se per attribuire un ruolo centrale alle abitudini, oppure per sottolineare il peso dei fattori innati ed eventualmente ereditari.

5. Ora, il problema può essere chiarito tenendo presente la situazione che era presupposta sia da Platone, sia da entrambe le Etiche di Aristotele. Esisteva infatti un'opinione comune, in Atene e in generale tra i Greci, secondo cui l'eccellenza umana (l'arete, parola che noi siamo soliti tradurre con "virtù") dipenderebbe da tre fattori: le doti naturali appunto, le abitudini che si assumono nella vita, la razionalità. Come l'opinione diffusa comunemente la presentano sia Platone (basta pensare alle prime parole del Menone), sia Aristotele (nelle Etiche e nella Politica) e sappiamo che era condivisa da alcuni sofisti, per lo meno Protagora, e da altre persone colte, come p. es. l'oratore Isocrate. ${ }^{10}$ All'interno di questa triade di fattori tradizionali della virtù si mosse la riflessione sia di Platone, sia di Aristotele, con opzioni differenti secondo le diverse loro opere. In Aristotele, come ho già detto, l'oscillazione e il cambiamento tra l'una e l'altra etica in merito al problema sono addirittura evidentissimi. Ma il problema di fondo è ancora quello: in qual senso avvenne il cambiamento e perché?

Una risposta abbastanza plausibile credo che la si ottenga riflettendo su quanto dice Platone nelle Leggi a proposito del rapporto tra la virtù fondata dalle abitudini e il piacere e il dolore. Dice appunto che il risultato delle buone abitudini assunte durante l'educazione giovanile è quello di riorientare le pulsioni naturali verso il piacere e il dolore dagli obiettivi naturali e fisici della mera conservazione della vita mediante il soddisfacimento dei bisogni fondamentali passando al piacere puramente psichico e mentale della virtù e all'avversione che devono ispirare il vizio e la malvagità. Ora, una simile enunciazione doveva essere una novità sconvolgente nella scuola di Platone: come risulta da allusioni di Aristotele in entrambe le Etiche, nell'Academia era infatti già corrente il collegamento di piacere e dolore con il tema della virtù, ma solo nel senso che piacere e dolore erano assunti come la materia bruta, il materiale grezzo, gli istinti diremmo forse noi, su cui la virtù sarebbe chiamata a esercitare il suo controllo: al punto che alcuni academici

10 I riferimenti ai testi possono essere visti nel mio libro citato alla nota 1, p. 26. 
giungevano a definire la virtù come "impassibilità verso il piacere e il dolore" (alla definizione Aristotele allude in entrambe le Etiche) ${ }^{11}{ }^{11}$ ' palese, credo, è anzi ovvio che chi così vede le cose non può nemmeno immaginare che ci sia un piacere della virtù a cui ci si potrebbe abituare fin da bambini. Ebbene, nell' $E E$ si dice sì che "la virtù riguarda piacere e dolore", ma poi si spiega questa proposizione solo nel senso veteroacademico del piacere e del dolore intesi come il materiale grezzo e bruto dal quale cautelarsi e su cui imporre un controllo; non si sa e non si dice mai, nell' $E E$, che si debba invece arrivare a una situazione in cui piacere e dolore dell'anima saranno addirittura gli indizi del conseguimento della virtù, come dice l'EN che lo ha imparato dalle Leggi, perché indicheranno il raggiungimento di una condizione psichica in cui si gioisce delle azioni virtuose e si prova dolore per lo spettacolo del vizio.

La mia conclusione è dunque questa, che l'EE deve essere stata scritta da Aristotele quando egli non conosceva ancora le Leggi di Platone (proprio come non le conoscevano i suoi colleghi academici che davano quella certa definizione della virtù); l'EN invece presuppone la conoscenza delle Leggi e implica un'attenta riflessione sulle novità che quest'ultima opera del maestro presentava. Grazie a essa, Aristotele aveva capito che, dopo e contro tanta insistenza sulle doti naturali, quale era stata quella che l'EE ancora ci mostra, c'era un altro modo di pensare lo sviluppo della virtù, un modo che implicava una maggior facilità, per gli uomini bene educati, di raggiungere quell'ideale di eccellenza morale.

Non abbiamo così una datazione assoluta di nessuna delle due Etiche e possiamo solo dire che l'EN deve essere posteriore al 348, ma non possiamo sapere di quanto; mentre dell' $E E$ possiamo dire soltanto che deve essere anteriore all'EN e alla pubblicazione delle Leggi, ma, di nuovo, non possiamo dire di quanto: non sappiamo quando le Leggi divennero note al pubblico e agli stessi scolari di Platone. Ma è molto più importante la possibilità, che spero si sia ottenuta, di capire almeno una delle ragioni forti che indussero Aristotele a sostituire l'EE con l'EN: da Platone e dalle Leggi aveva ancora imparato qualcosa di così importante da meritare di essere incluso nell'esposizione definitiva della teoria morale, che è l'Etica Nicomachea.

11 Ho lungamente discusso tutto il problema nel libro citato sopra, in nota 1, pp. 41-50. 